Logemann, Hartmut; Ilchmann, Achim :

An Adaptive Servomechanism for a Class of Infinite-Dimensional Systems

Zuerst erschienen in:

SIAM Journal on Control and Optimization 32 (1994) S. 917-936. DOI: $10.1137 / S 0363012991217482$ 


\title{
AN ADAPTIVE SERVOMECHANISM FOR A CLASS OF INFINITE-DIMENSIONAL SYSTEMS*
}

\author{
HARTMUT LOGEMANN $^{\dagger}$ AND ACHIM ILCHMANN ${ }^{\ddagger}$
}

\begin{abstract}
A universal adaptive controller is constructed that achieves asymptotic tracking of a given class of reference signals and asymptotic rejection of a prescribed set of disturbance signals for a class of multivariable infinite-dimensional systems that are stabilizable by high-gain output feedback. The controller does not require an explicit identification of the system parameters or the injection of a probing signal. In contrast to most of the work in universal adaptive control, this paper is based on an input-output approach and the results do not require a state-space representation of the plant. The abstract input-output results are applied to retarded systems and integrodifferential systems.
\end{abstract}

Key words. servomechanisms, adaptive control, high-gain feedback, infinite-dimensional systems, functional differential equations, input-output methods

AMS subject classifications. 34K20, 93B52, 93C25, 93C30, 93C 35, 93C40, 93D 15, 93D21, 93D25

1. Introduction. One of the most important applications of feedback is to achieve servoaction, that is, to obtain a closed-loop system that tracks a given class of reference signals and rejects a given class of external disturbances with zero asymptotic error. This problem has been well understood for many years provided that the plant is linear and time-invariant and the plant uncertainty is sufficiently small (see Wonham [30, p. 203] and Vidyasagar [27, p. 294] for finite-dimensional systems and Francis [4], Callier and Desoer [2], and Curtain [3] for infinite-dimensional systems). The basic design principle in the theory of linear servomechanisms, which is also referred to as the internal model principle, says (roughly speaking) that a controller that achieves robust servoaction necessarily contains a duplicate of the dynamics of the reference and disturbance signals.

If the plant uncertainty is large, which is the case if only certain structural information on the plant is available to the designer, it is desirable to construct a universal adaptive servomechanism, that is, a fixed nonlinear controller that achieves servoaction for a whole prescribed class of linear time-invariant systems and all possible initial conditions without explicit identification of the system parameters. Although the problem of universal adaptive stabilization of finite and infinite-dimensional systems has received considerable attention in recent years (cf. e.g., Mårtensson [17], [ 18], Logemann and Owens [14], Logemann and Mårtensson [13], and the references therein), there are only few papers on universal adaptive servomechanisms, which in addition deal exclusively with finite-dimensional systems. Mårtensson [19] pointed out that adaptive tracking of constant reference signals can be easily achieved for a given class of multivariable systems if a universal adaptive stabilizer is known and the class is invariant under precompensation by an integrator. Helmke, Prätzel-Wolters, and Schmid [8] proved a similar result for single-input single-output systems allowing for a more general class of reference signals including ramps, linear combinations of sinusoidal signals, etc. If the plant is known to lie in a given finite set of (multivariable) systems, if the reference and disturbance signals belong to the solution space of a given linear autonomous differential equation, and if an $L^{\infty}$-bound on the disturbances is known, Miller and Davison [21] constructed a switching controller that solves the servoproblem for any plant in this finite set. In [20] Miller and Davi-

* Received by the editors July 8, 1991; accepted for publication (in revised form) December 23, 1992.

$\dagger$ Institut für Dynamische Systeme, Fachbereich Mathematik/Informatik, Universität Bremen, Postfach 330440, 2800 Bremen 33, Germany. Current address, School of Mathematical Sciences, University of Bath, Bath BA2 2BZ, United Kingdom.

$\ddagger$ Centre for Systems and Control Engineering, School of Engineering, University of Exeter, Exeter, Devon EX4 $4 Q F$, United Kingdom. Current address, Institut für Angewandte Mathematik der Universität Hamburg, Bundesstr. 55,2000 Hamburg 13, Germany. 
son presented a low-gain controller that carries out asymptotic error regulation for constant reference and disturbance signals for any multivariable plant, provided it is asymptotically stable and has no transmission zeros at zero. For the class of all single-input single-output, relative degree one or two, minimum-phase systems of McMillan degree less than or equal to $n$, Morse [22] constructed a $4(n+1)$-dimensional model reference adaptive controller that achieves asymptotic tracking of any signal generated by a two-dimensional reference system. On the basis of high-gain concepts, Mareels [16] introduced a control law that solves the tracking problem for any single-input single-output minimum-phase system of known relative degree, provided the sign of the high-frequency gain and an upper bound on its magnitude is known. Finally, for the class of all single-input single-output minimum-phase systems having relative degree one, Helmke, Prätzel-Wolters, and Schmid [9] constructed a high-gain controller that has the property that the resulting closed-loop system tracks any reference signal annihilated by a given linear ordinary differential operator with constant coefficients.

The purpose of this paper is to construct a universal adaptive servomechanism for the class of multivariable infinite-dimensional systems that are minimum-phase and have an invertible high-frequency-gain. We show that the series interconnection of the controller presented in Byrnes and Willems [1] and a suitable precompensator solves the adaptive servoproblem for the class of systems under consideration. This result is also new for the finite-dimensional case. It generalizes the result in [9], where an adaptive tracking problem was solved for finitedimensional single-input single-output systems. The disturbance rejection problem is not addressed in [9]. Moreover, the proof in [9] does not extend to multivariable systems; neither does it carry over to infinite-dimensional plants, and so the generalization is far from being trivial. We mention that in [9] a state-space approach is used, while our treatment is based on the input-output set-up for high-gain adaptive stabilization as developed by Logemann and Owens [14]. So, in contrast to almost all papers in the area, our approach does not require a state-space model of the plant. Non-zero initial conditions are taken into account by using "initial-condition terms." The input-output results are applied to retarded systems and integrodifferential convolution systems.

The paper is organized as follows. In $\S 2$ we introduce a class of infinite-dimensional systems that are stabilizable by high-gain feedback and will be dealt with in the rest of the paper. Moreover, we collect a number of results on a functional differential equation of Volterra type that will be useful in what follows. Section 3 shows that the high-gain based switching algorithm, introduced by Byrnes and Willems [1] in a finite-dimensional state-space set-up, stabilizes any infinite-dimensional plant belonging to the class of systems introduced in $\$ 2$. Section 4 contains the main result of the paper. We prove that the series connection of the adaptive stabilizer presented in $\S 3$, followed by a suitable precompensator containing an internal model of the dynamics of the reference and disturbance signals, achieves servoaction for the class of systems under consideration. Section 5 is devoted to the application of the input-output results of $\xi 4$ to retarded systems and integrodifferential convolution systems. In particular it is shown that the adaptive servomechanism presented in $\S 4$ achieves "internal stability" in the sense that the internal variables of the plant and the precompensator remain bounded provided that the reference signal is bounded. The proof of a technical result is relegated to the Appendix.

\section{Nomenclature.}

$$
\begin{aligned}
\mathcal{C}_{+}:= & \text {open right-half plane. } \\
L L^{p}\left(\mathbb{H}_{+}, \mathbb{L}^{n}\right):= & \text { open left-half plane. } \\
& \text { vector space of locally } p \text {-integrable functions defined on } \mathbb{F}_{+} \\
& \text {with values in } \mathbb{R}^{n} .
\end{aligned}
$$


$H^{\infty}\left(\mathbb{C}^{n \times n}\right):=$ algebra of bounded holomorphic functions defined on $\mathbb{C}_{+}$ with values in $\mathbb{C}^{n \times n}$.

$H^{2}\left(\mathcal{C}^{n}\right):=$ the usual Hardy-Lebesgue space of order 2 of holomorphic functions defined on $\mathbb{C}_{+}$with values in $\mathbb{C}^{n}$.

$B V\left([a, b], \mathbb{F}:^{n \times n}\right):=$ vector space of $\mathbb{R}^{n \times n}$-valued functions of bounded variation defined on $[a, b]$.

$M\left(\mathbb{H}_{+}, \mathbb{F}_{-}^{n \times n}\right):=$ vector space of bounded Borel measures on $\mathbb{F}_{+}$with values in $\mathbb{F}^{n \times n}$.

Let $f$ be a function defined on $[0, a)$, where $0<a \leq \infty$. Then for all $\tau \in[0, a)$,

$$
\left(\pi_{\tau} f\right)(t):= \begin{cases}f(t), & 0 \leq t \leq \tau \\ 0, & t>\tau\end{cases}
$$

$\mathcal{L}$ denotes the Laplace transform.

The superscript " "” is used to denote Laplace transformed or Laplace-Stieltjes transformed functions.

2. Preliminaries and system description. We shall assume that externally our plant is described by a transfer-function matrix $G$ of size $m \times m$ which is meromorphic on $\mathbb{C}_{+}$and satisfies

$$
\left\{\begin{array}{l}
G^{-1}(s)=s D^{-1}+H(s) \\
\text { where } \quad D \in \mathbb{F}^{m \times m}, \operatorname{det}(D) \neq 0 \text { and } H \in H^{\infty}\left(\mathbb{C}^{m \times m}\right) .
\end{array}\right.
$$

Of course (2.1) is equivalent to

$$
G(s)=\left(I+\frac{1}{s} D H(s)\right)^{-1} \frac{1}{s} D,
$$

i.e., $G$ is the feedback interconnection of the integrator $(1 / s) D$ and the transfer-function matrix $H$.

In order to characterize condition (2.1) in terms of the zeros and the high-frequency behavior of $G$, we have to make precise what we mean by a zero of a meromorphic transferfunction matrix.

DEFINITION 2.1. Suppose that $R$ is a matrix of size $m \times m$ whose entries are meromorphic functions defined on a region $\Omega \subset C$. Let $(U, V)$ be a holomorphic right-coprime factorization of $R$ over $\Omega$, i.e., $U$ and $V$ are holomorphic matrices of size $m \times m$ defined on $\Omega$ such that $\operatorname{det}(V(s)) \not \equiv 0, R(s)=U(s) V^{-1}(s)$, and there exist holomorphic matrices $X$ and $Y$ of size $m \times m$ defined on $\Omega$ satisfying $X(s) U(s)+Y(s) V(s) \equiv I_{m} \cdot{ }^{\dagger}$ The zeros of $R(s)$ are defined to be the zeros of $\operatorname{det}(U(s))$.

PROPOSITION 2.2. Let $G(s)$ be a meromorphic transfer-function matrix of size $m \times m$ defined on a region $\Omega \supset \bar{C}_{+}$. Then $G^{-1}(s)$ admits a decomposition of the form $(2.1)$ if and only if

$$
\text { (i) } s G(s)-D=O(1 / s) \text { as }|s| \rightarrow \infty \text { in } \mathbb{C}_{+} \text {, }
$$

\footnotetext{
$\dagger$ Since the ring of holomorphic functions defined on a region has the property that finitely generated ideals are principal (see Rudin 23 , p. 328]) and since the field of meromorphic functions defined on a region is the quotient field of the ring of holomorphic functions defined on that region (see Rudin [23, p. 3271), it follows from Vidyasagar, Schneider, and Francis $[28]$ that such a factorization exists and is unique up to multiplication from the right by unimodular holomorphic matrices.
} 
and

(ii) $G(s)$ has no zeros in $\overline{\mathrm{C}_{+}}$.

Proof. See Logemann and Zwart [15].

Note that condition (i) in Proposition 2.2 is a generalization of the relative-degree one condition for finite-dimensional single-input single-output systems.

Remark 2.3. The transfer-function matrix $G$ of a stabilizable and detectable finitedimensional system $\dot{x}=A x+B u, y=C x$ satisfies (2.1) if and only if the system is minimum-phase, i.e.,

$$
\operatorname{det}\left(\begin{array}{cr}
s I-A & -B \\
C & 0
\end{array}\right) \neq 0 \text { for all } s \in \overline{\mathbb{C}}_{+},
$$

and has invertible high-frequency gain, i.e., $\operatorname{det}(C B) \neq 0$. Moreover the matrix $D$ in $(2.1)$ is given by $C B$.

In the following we shall assign an operator $\mathcal{H}: L^{2}\left(\mathbb{E}_{+}, \mathbb{C}^{m}\right) \rightarrow L^{2}\left(\mathbb{E}_{+}, \mathbb{C}^{m}\right)$ to the transfer-function matrix $H$ by defining $\mathcal{H}:=\mathcal{L}^{-1} \mathcal{M}_{H} \mathcal{L}$, where $\mathcal{L}$ denotes the Laplace transform and $\mathcal{M}_{H}$ denotes the multiplication by $H$ on the Hardy space $H^{2}\left(\mathbb{C}^{m}\right)$. The operator $\mathcal{H}$ is linear, bounded, and shift-invariant (in the sense of Vidyasagar [26]). As a consequence $\mathcal{H}$ is causal (see [26]) and therefore has a unique causal extension to $L L^{2}\left(\mathbb{F}_{+}, \mathbb{C}^{m}\right)$. This extension will also be denoted by $\mathcal{H}$. The converse is also true, i.e., given a linear, bounded, shift-invariant operator $\mathcal{H}: L^{2}\left(\mathcal{F}_{-}, \mathbb{C}^{m}\right) \rightarrow L^{2}\left(\mathbb{K}_{+}^{+}, \mathbb{C}^{m}\right)$, there exists $H \in H^{\infty}\left(\mathbb{C}^{m \times m}\right)$ such that $\mathcal{H}=\mathcal{L}^{-1} \mathcal{M}_{H} \mathcal{L}$ (see Harris and Valenca [7], Logemann [12], and Weiss [29]). Finally we mention that $L L^{2}\left(\mathbb{E}_{+}, \mathbb{F}^{m}\right)$ is an $\mathcal{H}$-invariant subspace of $L L^{2}\left(\mathbb{R}_{+}, \mathbb{C}^{m}\right)^{\ddagger}$ if and only if $H(s)=\bar{H}(\bar{s})$ for all $s \in C_{+}$. In control applications the latter condition will always be satisfied and it is assumed to hold in the following.

The function $G$ satisfying (2.1) can be thought of as being the transfer-function matrix of

$$
\dot{y}=D(u-(\mathcal{H} y+w)), \quad y(0)=y_{0} \in \mathbb{P}^{m},
$$

where $u \in L L^{1}\left(\mathbb{F}_{+}, \mathbb{F}_{-}^{m}\right)$ and $w \in L^{2}\left(\mathbb{F}_{+}, \mathbb{F}^{m}\right)$ takes account of non-zero initial conditions in the system with transfer-function matrix $H$. The initial value problem (2.3) is a special case of the following initial value problem, which will play an important role in this paper. Consider

$$
\begin{aligned}
\dot{x}(t) & =(S x)(t)+f(t, x(t))+g(t), \quad t \geq \alpha, \quad, \quad \alpha \geq 0, \\
x_{\left.\right|_{[0, \alpha]}} & =x_{0} \in C\left([0, \alpha], \mathbb{E}^{n}\right), \quad
\end{aligned}
$$

where the following hold.

(i) $S: L L^{2}\left(\mathbb{L}_{+}, \mathbb{F}_{-}^{n}\right) \rightarrow L L^{2}\left(\mathbb{F}_{+}, \mathbb{E}^{n}{ }^{n}\right)$. We assume that $S(0)=0$ and that there exists $\kappa>0$ such that $\left\|\pi_{t}\left(S x-S x^{\prime}\right)\right\| \leq \kappa_{\|}\left\|\pi_{t}\left(x-x^{\prime}\right)\right\|$ for all $x, x^{\prime} \in L L^{2}\left(\mathbb{F}_{-}^{\prime}, \mathbb{F}^{\prime n}\right)$ and for all $t \geq 0$, i.e., $S$ is unbiased, causal, and of finite incremental gain.

(ii) $f: \mathbb{F}_{+} \times \mathbb{F}^{n} \rightarrow \mathbb{F}_{-}^{n}$ is a function. We assume that $f(t, x)$ is continuous in $t$ and locally Lipschitz continuous in $x$, uniformly in $t$ on bounded intervals.

(iii) $g$ is in $L L^{1}\left(\mathbb{F i}^{+}, H^{n}\right)$.

Of course, if $\alpha=0$ in (2.4), then $\left.\left.C([0, \alpha],]^{n}\right)=\right]^{n}$. In order to define what we mean by a solution of the initial value problem (2.4) on $[0, \beta)(\alpha<\beta \leq \infty)$, we have to give a meaning to $S x$ if $x \in C\left([0, \beta)\right.$, 识 $\left.{ }^{n}\right)$ (remember that $S$ operates on functions whose domain of definition is $\left.H_{+}\right)$. We set $(S x)(t)=\left(S \pi_{\tau} x\right)(t)$ for $0 \leq t \leq \tau<\beta$. Since $S$ is causal, this definition does not depend on the choice of $\tau$.

\footnotetext{
$\ddagger$ Notice that here $L L^{2}\left(\mathbb{P}_{+}, c^{m}\right)$ is considered as a real vector space.
} 
DEFINITION 2.4. A solution of (2.4) on $[0, \beta)(\alpha<\beta \leq \infty)$ is an absolutely continuous function $x$ on $[0, \beta)$ such that $x_{\mid[0, \infty]}=x_{0}$ and the differential equation in (2.4) is satisfied by $x$ almost everywhere on $[0, \beta)$.

THEOREM 2.5. The initial-value problem (2.4) has a unique solution on some interval $[0, \beta)$, where $\alpha<\beta \leq \infty$. If $\beta<\infty$ and $\beta$ cannot be increased, then there exists a strictly increasing sequence $t_{i} \in(0, \beta)$, satisfying $\lim _{i \rightarrow \infty} t_{i}=\beta$, such that $\lim _{i \rightarrow \infty}\left\|x\left(t_{i}\right)\right\|=\infty$.

The above theorem has been proved in Logemann and Owens [14]. Similar results can be found in Gripenberg, Londen, and Staffans [5, p. 359], and Hinrichsen and Pritchard [10]. Theorem 2.5 implies in particular that the initial value problem (2.3) has a unique solution for all $w \in L^{2}\left(\mathbb{F}_{+}^{\prime}, \mathbb{H}^{\prime m}\right), u \in L L^{\mathbf{l}}\left(\mathbb{H}_{+}, \mathbb{H} !^{m}\right)$, and $y_{0} \in \mathbb{H}^{m}$.

3. Adaptive stabilization. The aim of this section is to construct a universal adaptive control law that stabilizes any system of the form (2.3), i.e., the control law does not depend on $D$ and $\mathcal{H}$, and the closed-loop system satisfies $\lim _{t \rightarrow \infty} y(t)=0$ for all $y_{0} \in \mathbb{K}^{m}$ and $w \in L^{2}\left(\mathbb{F}_{+}, \mathbb{L}_{-}^{m}\right)$.

In the following, we need a result from linear algebra which has been proved by Mårtensson [17], [18]. For $m \geq 1$ we call a set $\mathcal{U} \subset G L(m,[\mathbb{L})$ unmixing, if for any $A \in G L(m, 1+)$ there is a $U \in \mathcal{U}$ such that $\operatorname{spec}(A U) \subset \mathbb{U}_{-}$.

PROPOSITION 3.1 ([17], [18]). For all $m \geq 1$, there exist unmixing sets of finite cardinality.

Unfortunately the cardinality of the unmixing sets constructed in [17], [18] is far too large than would be convenient for applications. Hardly anything is known on the minimum cardinality of unmixing sets. However, for $m=1$ the set $\{1,-1\}$ is obviously unmixing, while for $m=2$ there exists an unmixing set of cardinality 6 (see [17], [18]). It has been shown by Zhu [31] that $G L(3,1)$ can be unmixed by a set having cardinality 32 .

In the following, let $\left\{K_{1}, \ldots, K_{N}\right\}$ be an unmixing set for $G L\left(m, \mathbb{F}_{-}\right)$. Since (2.3) can be stabilized by high-gain feedback of the form $u(t)=k y(t)$, provided that $\operatorname{spec}(D) \subset{ }_{-}$ and $k$ is a sufficiently large positive number, it seems reasonable to consider the following adaptive control law:

$$
\begin{aligned}
& u(t)=k(t) K_{\sigma(k(t))} y(t), \\
& \dot{k}(t)=\|y(t)\|^{2}, \quad k(0)=k_{0} \in \mathbb{E} .
\end{aligned}
$$

In (3.1) the function $\sigma: \mathbb{P}-\rightarrow\{1, \ldots, N\}$ is given by

$$
\sigma(k)= \begin{cases}1, & k \in\left[-\tau_{\mathbf{l}}, \tau_{1}\right), \\ i, & k \in\left[\tau_{l N+i}, \tau_{l N+i+1}\right) \cup\left[-\tau_{l N+i+1},-\tau_{l N+i}\right) \text { for some } l \in \mathrm{I}_{0},\end{cases}
$$

where the sequence $\left(\tau_{j}\right)_{j \in \mathrm{H}_{0}}$ is defined as

$$
\tau_{j+1}=\tau_{j}^{2}, \quad \tau_{1}>1 .
$$

Note that the gain $k(t)$ is monotonically increasing and thus the function $\sigma$ ensures that $K_{\sigma(k(t))}$ will hit some stabilizing gain matrix $K_{i}$ if $k(t)$ diverges. The growth condition (3.3) captures the intuitive idea that the length of the intervals $\left[\tau_{j}, \tau_{j+1}\right)$ should increase rapidly, in order to enable the closed-loop system to settle down. Although the closed-loop system given by (2.3) and (3.1) is of the form (2.4), we cannot apply Theorem 2.5 straight away in order to establish well posedness of the closed loop, since the map $\mathbb{F} \rightarrow\left\{K_{1}, \ldots, K_{N}\right\}, k \mapsto K_{\sigma(k)}$ is not continuous. However, Theorem 2.5 can be used to prove the following.

LEMMA 3.2. For each pair of initial conditions $\left(y_{0}, k_{0}\right) \in \mathbb{R}^{m} \times \mathbb{w}_{\text {. }}$ and for each $w \in$ $L^{2}\left(\mathbb{L}_{+}, \mathbb{Z}^{m}\right)$, the closed-loop system given by (2.3) and (3.1) has a unique absolutely continuous solution $(y, k)$ that can be extended to the right as long as it remains bounded. 
Proof. See Appendix.

Now we are in the position to prove the main result of this section. It says that the control law (3.1) stabilizes any system of the form (2.3), or in other words (3.1) is a universal adaptive control law for this class.

THEOREM 3.3. The solution $(y, k)$ of the closed-loop system given by (2.3) and (3.1) exists on $1 \mathrm{H}+$ and has the following properties:

(i) $\lim _{t \rightarrow \infty} k(t)$ exists and is finite;

(ii) $y \in L^{2}\left(\mathbb{F}_{+}^{+}, \mathbb{E}^{m}\right) \cap L^{\infty}\left(\mathbb{F}_{+}, \mathbb{F}^{m}\right)$;

(iii) $\lim _{t \rightarrow \infty} y(t)=0$.

We shall prove Theorem 3.3 by combining ideas of Byrnes and Willems [1] with the following lemma, which can be found in Ilchmann and Logemann [11].

LEMMA 3.4. Suppose that $\sigma$ and $\tau_{j}$ are given by (3.2) and (3.3), respectively, and for $\alpha>0$ and $i \in\{1, \ldots, N\}$ define $F_{i}^{\alpha}: \mathbb{F} \rightarrow\{1,-\alpha\}$ by

$$
F_{i}^{\alpha}(x)=\left\{\begin{aligned}
1, & \text { if } \sigma(x)=i \\
-\alpha, & \text { if } \sigma(x) \neq i
\end{aligned}\right.
$$

Then we have

$$
\sup _{k>k_{0}} \frac{1}{k-k_{0}} \int_{k_{i 1}}^{k} x F_{i}^{\alpha}(x) d x=+\infty
$$

for all $k_{0} \in \mathbb{R}, \alpha>0, i \in\{1, \ldots, N\}$.

Proof of Theorem 3.3. By assumption there exists $i \in\{1, \ldots, N\}$ such that $\operatorname{spec}\left(D K_{i}\right) \subset C_{-}$. Hence there is a positive definite matrix $Q=Q^{T} \in G L(m$, IF: satisfying

$$
K_{i}^{T} D^{T} Q+Q D K_{i}=-I
$$

Furthermore, choose $\alpha>0$ such that

$$
K_{j}^{T} D^{T} Q+Q D K_{j} \leq \alpha I \text { for all } j \in\{1, \ldots, N\} .
$$

By Lemma 3.2, the closed-loop system given by (2.3) and (3.1) has a unique solution $(y, k)$. Let $\left[0, t^{*}\right)$ denote its maximal interval of existence. Setting $\|x\|_{Q}:=(\langle x, Q x\rangle)^{1 / 2}$ for $x \in \mathbb{R}: m$ and using (2.3), (3.4), (3.6), and (3.7) we obtain

$$
\begin{aligned}
\frac{d}{d t}\|y(t)\|_{Q}^{2}= & \dot{y}(t)^{T} Q y(t)+y(t)^{T} Q \dot{y}(t) \\
= & k(t) y(t)^{T}\left(K_{\sigma(k(t))}^{T} D^{T} Q+Q D K_{\sigma(k(t))}\right) y(t)-(\mathcal{H} y)(t)^{T} D^{T} Q y(t) \\
& -w(t)^{T} D^{T} Q y(t)-y(t)^{T} Q D(\mathcal{H} y)(t)-y(t)^{T} Q D w(t) \\
\leq & -F_{i}^{\alpha}(k(t)) k(t) \dot{k}(t)-2 y(t)^{T} Q D(\mathcal{H} y)(t)-2 y(t)^{T} Q D w(t) .
\end{aligned}
$$

Using Hölder's inequality and the causality and boundedness of $\mathcal{H}$, it is easy to show that for all $f \in L L^{2}\left(\mathbb{R}+, \mathbb{C}^{m}\right)$ and $t \geq 0$,

$$
\left|\int_{0}^{t} f(\tau)^{T} Q D(\mathcal{H} f)(\tau) d \tau\right| \leq\|Q\|\|D\|\|\mathcal{H}\| \int_{0}^{t}\|f(\tau)\|^{2} d \tau
$$


Integrating (3.8) from 0 to $t, t<t^{*}$, changing variables, and applying (3.9) yields

$$
\begin{aligned}
\|y(t)\|_{Q}^{2}-\left\|y_{0}\right\|_{Q}^{2} \leq & -\int_{k_{0}}^{k(t)} x F_{i}^{\alpha}(x) d x+\lambda_{1} \int_{0}^{t}\|y(\tau)\|^{2} d \tau \\
& +\lambda_{2}\|w\|_{2}\left(\int_{0}^{t}\|y(\tau)\|^{2} d \tau\right)^{1 / 2} \\
= & \left(k(t)-k_{0}\right)\left\{\lambda_{1}+\frac{\lambda_{2}\|w\|_{2}}{\sqrt{k(t)-k_{0}}}-\frac{1}{k(t)-k_{0}} \int_{k_{1}}^{k(t)} x F_{i}^{\alpha}(x) d x\right\},
\end{aligned}
$$

where $\lambda_{1}$ and $\lambda_{2}$ are suitable positive constants depending on $\mathcal{H}, D$, and $Q$.

In order to show global existence of the solution $(y, k)$ on $\mathbb{F}_{+}$it is (by Lemma 3.2) sufficient to show that $(y, k)$ is bounded on $\left[0, t^{*}\right)$. In order to prove that $k(t)$ is bounded on $\left[0, t^{*}\right)$, assume the contrary. It then follows from Lemma 3.4 that the limes inferior of the right-hand side of (3.10) is $-\infty$, contradicting the fact that the left-hand side of $(3.10)$ is bounded from below by $-\left\|y_{0}\right\|_{Q}^{2}$. Hence $k(t)$ is bounded on $\left[0, t^{*}\right)$ and from (3.1) and (3.10) we obtain that $y \in L^{2}\left(0, t^{*} ; \mathbb{Z}^{m}\right) \cap L^{\infty}\left(0, t^{*} ; \mathbb{F}_{-}^{m}\right)$. In particular we have $t^{*}=\infty$, which implies (i) and (ii). In order to prove (iii), notice that by (2.3), (i), and (ii) $\dot{y} \in L^{2}\left(\mathbb{E}_{+} ; \mathbb{R}^{m}\right)$. As a consequence (iii) holds true.

Remark 3.5. (i) It is not difficult to see that the sequence given by (3.3) can be replaced by any strictly increasing sequence $\left(\tau_{j}\right)_{j \in \mathbb{A}}$ satisfying $\lim _{j \rightarrow \infty} \tau_{j} / \tau_{j-1}=+\infty$ (cf. Ilchmann and Logemann [11] and Ryan [24]).

(ii) Let $u \in L L^{2}\left(\mathbb{E}_{+}^{+}, \mathbb{F}_{+}^{m}\right), w \in L^{2}\left(\mathbb{F}_{+}^{+}, \mathbb{F}_{+}^{m}\right)$ and suppose that $y$ satisfies (2.3). If $Q \in \mathbb{R}^{m \times m}$ is positive definite, then the inequality

$$
\|y(t)\|_{Q}^{2} \leq\left\|y_{0}\right\|_{Q}^{2}+\mu\left(1+\int_{0}^{t}\|y(\tau)\|^{2} d \tau\right)+2 \int_{0}^{t} y(\tau)^{T} Q D u(\tau) d \tau
$$

holds for all $t \geq 0$, where $\mu$ is a suitable positive constant depending on $\mathcal{H}, D, Q$, and $w$. Inequality (3.11) has been derived implicitly in the proof of Theorem 3.3 and may be of some independent interest.

Remark 3.6. The controller (3.1) was introduced by Byrnes and Willems [1] in a finitedimensional state-space set-up. The main result in [1] says that any finite-dimensional statespace system with $m$ inputs and $m$ outputs can be stabilized by the control law (3.1), provided it is minimum-phase and has invertible high-frequency gain. However, the proof is not convincing, since the inequality (3.4) in [1] is in general wrong. A result similar to that in [1] can be found in Mårtensson [17], [18]. The proof in [17], [18] is not convincing either, since it is based on the claim that for the adaptive control system

$$
\begin{aligned}
& \dot{x}(t)=A x(t)+B u(t), \quad x(0)=x_{0} \in \mathbb{F}^{n}, \\
& y(t)=C x(t), \\
& u(t)=k(t) Q y(t), \\
& \dot{k}(t)=\|u(t)\|^{2}+\|y(t)\|^{2}, \quad k(0)=k_{0} \in \mathbb{E},
\end{aligned}
$$

there exist constants $c>0$ and $T \geq 0$ such that

$$
\int_{t}^{\infty}\left(\|u(\tau)\|^{2}+\|y(\tau)\|^{2}\right) d \tau \leq c\|x(t)\|^{2}
$$

for all $x_{0} \in \mathbb{F}^{n}, k_{0} \in \mathbb{F}, t \geq T$, provided that $(A, B, C)$ is minimum-phase and $\sigma(C B Q) \subset \mathbb{C}_{-}$. 


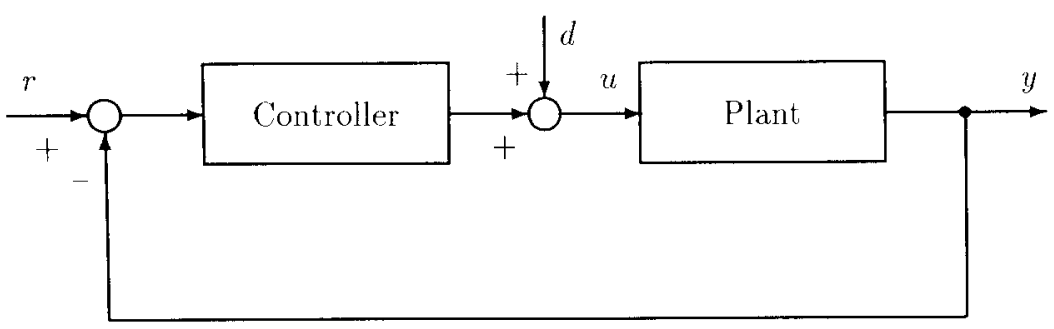

FIG. 1. Closed-loop sytem.

This is not proved in [17], [18] and it seems to the authors that it is unlikely to hold true.

We close this section with a conjecture on the limiting closed-loop system. If the assumptions of Theorem 3.3 are satisfied, then $\lim _{t \rightarrow \infty} k(t)=: k_{\infty}\left(w, y_{0}, k_{0}\right)$ exists and is finite. The linear system

$$
\left\{\begin{array}{l}
\dot{\tilde{y}}=D\left[k_{\infty}\left(w, y_{0}, k_{0}\right) K_{\sigma\left(k_{\infty}\left(w, y_{0}, k_{0}\right)\right)} \tilde{y}-\mathcal{H} \bar{y}-\bar{w}\right] \\
\tilde{y}(0)=\tilde{y}_{0} \in \mathbb{E}^{m}, \tilde{w} \in L^{2}\left(\mathbb{E}_{+}, \mathbb{E}^{m}\right)
\end{array}\right.
$$

is called the terminal system of the nonlinear closed-loop system given by (2.3) and (3.1). It is easy to see that (3.12) does not satisfy $\lim _{t \rightarrow \infty} \tilde{y}(t)=0$ for arbitrary $\left(\tilde{w}, \tilde{y}_{0}\right) \in L^{2}\left(\mathbb{H}_{+}, \mathbb{F}_{-}^{m}\right) \times$ $\mathbb{I}_{1}{ }^{m}$. Indeed, consider the special case that $\mathcal{H}=0$ and choose $w=0, y_{0}=0$, and $k_{0}=0$ in (2.3) and (3.1). Since $k_{\infty}(0,0,0)=0$, it follows that the solution $\tilde{y}$ of (3.12) is given by $\tilde{y}(t)=\bar{y}_{0}-D \int_{0}^{t} \tilde{w}(\tau) d \tau$, and hence $\tilde{y}(t)$ in general does not converge to 0 as $t \rightarrow \infty$. However, recent work of Townley [25] on adaptive stabilization of finite-dimensional systems leads us to the following conjecture.

Conjecture. For given $k_{0} \in \mathbb{1}$. there exists an open and dense set $\mathcal{I}\left(k_{0}\right) \subset L^{2}\left(\bar{T}_{+}, \mathbb{H}^{m}\right) \times$ $\mathbb{F}^{m}{ }^{m}$ such that the terminal system (3.12) is stable in the sense that

$$
\left.\tilde{y} \in L^{2} \cap L^{\infty}\left(\mathbb{F}_{+}^{\prime}, w^{m}\right) \text { and } \lim _{t \rightarrow \infty} \tilde{y}(t)=0 \text { for all }\left(\tilde{w}, \tilde{y}_{0}\right) \in L^{2}\left(\mathbb{F}_{+}, \mathbb{F}^{m}\right) \times\right] \mathrm{H}^{m} \text {, }
$$

provided that $\left(w, y_{0}\right) \in \mathcal{I}\left(k_{0}\right)$.

4. Adaptive tracking and disturbance rejection. Consider the control scheme in Fig. 1, where the plant is described by $(2.1)$ or, equivalently, by (2.3). The aim of this section is to construct a single controller, such that the closed-loop system asymptotically tracks a given reference trajectory $r$ and asymptotically rejects a given disturbance signal $d$ for all plants of the form (2.1). The signals $r$ and $d$ belong to prespecified vector spaces of functions that are defined as follows. Let $\rho_{i}, \delta_{i} \in \mathbb{E}:[s]$ be monic polynomials, $1 \leq i \leq m$, and set $\rho:=\left(\rho_{1}, \ldots, \rho_{m}\right)^{T}$ and $\delta:=\left(\delta_{1}, \ldots, \delta_{m}\right)^{T}$. The admissible reference signals are given by

$$
\mathcal{S}_{\rho}:=\left\{r: \mathbb{F}_{+} \rightarrow \mathbb{F}_{-}^{m} \mid \rho_{i}\left(\frac{d}{d t}\right) r_{i} \equiv 0, i=1, \ldots, m\right\},
$$

while the disturbances $d$ are supposed to belong to $\mathcal{S}_{\delta}+L^{2}\left(\mathrm{~J}_{+}, \mathrm{I}_{-} m\right)$, where $\mathcal{S}_{\delta}$ is defined as $\mathcal{S}_{\rho}$ with $\rho_{i}$ replaced by $\delta_{i}$. The well-known internal model principle from linear control theory (see e.g., Wonham [30, p. 203], and Vidyasagar [27, p. 294], for the finite-dimensional case and Francis [4], Callier and Desoer [2], and Curtain [3] for the infinite-dimensional case) suggests that the dynamics of the reference and disturbance signals should be replicated in the 


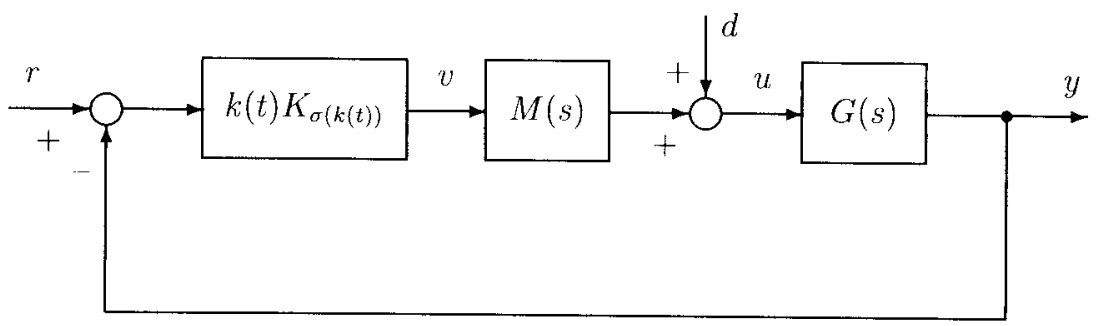

FIG. 2. High-gain adaptive senomechanism.

loop via a precompensator. To this end set

$$
p(s)=\operatorname{lcm}\left(s, \rho_{1}(s), \ldots, \rho_{m}(s), \delta_{1}(s), \ldots, \delta_{m}(s)\right),
$$

where we choose $p$ to be monic. Moreover, let $q$ be a monic polynomial that is Hurwitz and satisfies $\operatorname{deg}(q)=\operatorname{deg}(p)$. We define the precompensator $M(s)$ containing the internal model to be

$$
M(s)=\frac{q(s)}{p(s)} I_{m}
$$

Note that by construction $M(s)$ contains an integrator. This is required for a purely technical reason: Without $M(s)$ having a pole in 0 we were not able to prove Theorem 4.1 below. Let $G_{M}$ denote the precompensated plant, i.e., $G_{M}(s)=G(s) M(s)$. Now realize that, by $(2.1)$,

$$
G_{M I}^{-1}(s)=\frac{p(s)}{q(s)}\left(s D^{-1}+H(s)\right)=s D^{-1}+H_{M}(s)
$$

where

$$
H_{M}(s):=s\left(\frac{p(s)}{q(s)}-1\right) D^{-1}+\frac{p(s)}{q(s)} H(s)
$$

belongs to $H^{\infty}\left(0^{m \times m}\right)$. The important point here is that the structural property $(2.1)$ of the plant $G$ remains invariant under precompensation by $M(s)$. The overall adaptive controller we shall investigate in the following is given by

$$
\left\{\begin{aligned}
\hat{u}(s) & =M(s) \hat{v}(s)+\hat{d}(s), \\
v(t) & =k(t) K_{\sigma(k(t))}(r(t)-y(t)), \\
\dot{k}(t) & =\|r(t)-y(t)\|^{2}, \quad k(0)=k_{0},
\end{aligned}\right.
$$

where $\sigma$ and $K_{1}, \ldots, K_{N}$ are defined as in $\xi 3$ (cf. Fig. 2). Using the fact that the first equation of (4.2) can be written as

$$
\hat{u}(s)=M(s)\left(\hat{u}(s)+M^{-1}(s) \hat{d}(s)\right),
$$

setting $d_{M}(t):=\mathcal{L}^{-1}\left(M^{-1} \hat{d}\right)(t)$ and $\mathcal{H}_{M}:=\mathcal{L}^{-1} \mathcal{M}_{H_{\Lambda}} \mathcal{L}{ }^{\dagger}$ we obtain the following timedomain description of the closed-loop system given by (2.3) and (4.2):

\footnotetext{
$\dagger$ As before, the unique causal extension of $\mathcal{H}_{M}$ to $L L^{2}\left(\mathbb{F}_{+}, \mathbb{E}^{m}\right)$ will be denoted by the same symbol $\mathcal{H}_{M}$
} 


$$
\left\{\begin{array}{l}
\dot{y}(t)=D\left(v(t)+d_{M}(t)-\left(\mathcal{H}_{M} y\right)(t)-w_{M}(t)\right), \quad y(0)=y_{0}, \quad w_{M} \in L^{2}\left(\mathbb{H}_{+}, \mathbb{P}^{m}\right) \\
v(t)=k(t) K_{\sigma(k(t))}(r(t)-y(t)) \\
\dot{k}(t)=\|r(t)-y(t)\|^{2}, \quad k(0)=k_{0},
\end{array}\right.
$$

where as in $\S 2$ the term $w_{M}$ takes account of non-zero initial conditions (cf. also $\S 5$ ).

We are now in the position to prove the main result of this paper, which shows that the controller (4.2) solves the servoproblem for all systems of the form (2.3).

THEOREM 4.1. The solution $(y, k)$ of the closed-loop system (4.3) exists on $\mathbb{F}_{+}$and has the following properties:

(i) $\lim _{t \rightarrow \infty} k(t)$ exists and is finite,

(ii) $y-r \in L^{2}\left(\mathbb{F}_{+}^{+}, \mathbb{L}^{m}\right) \cap L^{\infty}\left(\mathbb{E}_{+}, \mathbb{E}_{-}^{m}\right)$,

(iii) $\lim _{t \rightarrow \infty}(y(t)-r(t))=0$.

Proof. Rewriting the first equation of (4.3) as

$$
\frac{d}{d t}(r-y)=-D\left(v+\mathcal{H}_{M}(r-y)+d_{M}-\mathcal{H}_{M} r-D^{-1} \dot{r}-w_{M}\right)
$$

we see that (i)-(iii) will follow from Theorem 3.3, provided that the term $d_{M}-\mathcal{H}_{M} r-D^{-1} \dot{r}$ belongs to $L^{2}\left(\mathbb{F}_{+}^{\prime}, \mathbb{H}^{m}\right)$. It is easy to show that $d_{M} \in L^{2}\left(\mathbb{F}_{+}^{\prime}, \mathbb{R}^{m}\right)$. Indeed, by definition we have

$$
\hat{d}_{M}(s)=M^{-1}(s) \hat{d}(s)=M^{-1}(s) \hat{d}_{1}(s)+M^{-1}(s) \hat{d}_{2}(s)
$$

where $d_{1} \in L^{2}\left(\mathbb{F}_{+}, \mathbb{F}^{m}\right)$ and $d_{2} \in \mathcal{S}_{\delta}$. Now, clearly we have

$$
M^{-1}(s) \hat{d}_{1}(s) \in H^{2}\left(\mathbb{C}^{m}\right) \text {. }
$$

Moreover, since $p(d / d t) d_{2 i} \equiv 0,1 \leq i \leq m$ (where $d_{2 i}$ denotes the $i$ th component of $d_{2}$ ), it follows that there exist polynomials $\beta_{i} \in \mathbb{E}:[s]$ such that

$$
\hat{d}_{2 i}(s)=\frac{\beta_{i}(s)}{p(s)} \quad \text { and } \quad \operatorname{deg}\left(\beta_{i}\right) \leq \operatorname{deg}(p)-1=\operatorname{deg}(q)-1 .
$$

Therefore

$$
M^{-1}(s) \hat{d}_{2}(s)=\left(\frac{\beta_{1}(s)}{q(s)}, \ldots, \frac{\beta_{m}(s)}{q(s)}\right)^{T} \in H^{2}\left(c^{m}\right) .
$$

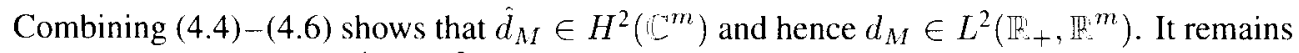
to show that $\mathcal{H}_{M} r+D^{-1} \dot{r} \in L^{2}\left(\mathbb{F}_{+}, \mathbb{W}^{m}\right)$. This will be done in two steps.

Step 1. Suppose that $r(0)=0$. Then we have

$$
\mathcal{L}\left(\mathcal{H}_{M} r+D^{-1} \dot{r}\right)(s)=H_{M}(s) \hat{r}(s)+s D^{-1} \hat{r}(s),
$$

and moreover $\hat{r}(s)=[1 / p(s)] \gamma(s)$, where $\gamma(s):=\left(\gamma_{1}(s), \ldots, \gamma_{m}(s)\right)^{T}, \gamma_{i} \in \mathbb{R}:[s]$, and $\operatorname{deg}\left(\gamma_{i}\right) \leq \operatorname{deg}(p)-2=\operatorname{deg}(q)-2$. Using (4.1) it follows from (4.7) that

$$
\begin{aligned}
\mathcal{L}\left(\mathcal{H}_{M} r+D^{-1} \dot{r}\right)(s)= & s\left(\frac{p(s)}{q(s)}-1\right) D^{-1} \frac{1}{p(s)} \gamma(s) \\
& +\frac{p(s)}{q(s)} H(s) \frac{1}{p(s)} \gamma(s)+s D^{-1} \frac{1}{p(s)} \gamma(s) \\
= & D^{-1} \frac{s}{q(s)} \gamma(s)+H(s) \frac{1}{q(s)} \gamma(s) \in H^{2}\left(\mathbb{C}^{m}\right),
\end{aligned}
$$


since $H \in H^{\infty}\left(\mathbb{C}^{m \times m}\right), \operatorname{deg}\left(\gamma_{i}\right) \leq \operatorname{deg}(q)-2$ and $q$ is Hurwitz. Hence we have shown that

$$
\mathcal{H}_{M} r+D^{-1} \dot{r} \in L^{2}\left(\mathbb{F}_{+}, \mathbb{F}_{-}^{m}\right),
$$

provided that $p(d / d t) r \equiv 0$ and $r(0)=0$.

Step 2. Now suppose that $r(0)=r_{0} \neq 0$. Define $z(t):=r(t)-\Theta(t) r_{0}$, where

$$
\Theta(t):= \begin{cases}0, & t<0 \\ 1, & t \geq 0\end{cases}
$$

notice that

$$
\mathcal{H}_{M} r+D^{-1} \dot{r}=\mathcal{H}_{M} z+D^{-1} \dot{z}+\mathcal{H}_{M}\left(\Theta r_{0}\right)
$$

Since $p(0)=0$, it follows that $p(d / d t) z \equiv 0$. Moreover $z(0)=0$ and hence we obtain from Step 1 that $\mathcal{H}_{M} z+D^{-1} \dot{z} \in L^{2}\left(\mathbb{F}_{+}, \mathbb{F}^{m}\right)$. Therefore, by (4.8) it remains to show that $\mathcal{H}_{M}\left(\Theta r_{0}\right) \in L^{2}\left(\mathrm{~F}_{+}, \mathbb{P}^{m}\right)$. To this end write $p(s)=s \tilde{p}(s), \tilde{p} \in \mathbb{E}[s]$, which is possible by assumption. Using (4.1) it follows that

$$
\mathcal{L}\left(\mathcal{H}_{M}\left(\Theta r_{0}\right)\right)(s)=H_{M}(s) \underset{s}{\frac{1}{s}} r_{0}=\left(\frac{p(s)}{q(s)}-1\right) D^{-1} r_{0}+\frac{\tilde{p}(s)}{q(s)} H(s) r_{0} \in H^{2}\left(\mathbb{C}^{m}\right)
$$

and hence $\mathcal{H}_{M}\left(\Theta r_{0}\right) \in L^{2}\left(\mathbb{F}_{+}^{\prime}, \mathbb{F}^{m}\right)$.

5. Applications to retarded systems and integrodifferential convolution systems. In this section we show how retarded and integrodifferential convolution systems fit into the input-output set-up developed in $\S \S 3$ and 4 . We solve the adaptive servoproblem for these classes of systems under the assumption that the plant is minimum-phase and has invertible high-frequency gain. Moreover, it turns out that the internal variables of the plant and the precompensator remain bounded, provided that the reference signal is bounded.

5.1. Retarded systems. In the following we extend any function $F \in B V\left([a, b],\left[\mathbb{F}^{n \times n}\right)\right.$ to the whole real axis by setting $F(t)=F(a)$ for $t<a$ and $F(t)=F(b)$ for $t>b$. Any measurable function $f: \Omega \rightarrow \mathbb{E}^{n}, \Omega \subset \mathbb{R}$, will be extended to the whole real axis by defining $f(t)=0$ for $t \notin \Omega$. For $F=\left(F_{i j}\right) \in B V\left([0, h], \mathbb{F}_{-}^{n \times n}\right)$ and $f=\left(f_{1}, \ldots, f_{n}\right)^{T}, f_{i} \in$ $L L^{1}\left(\mathbb{F}, \mathbb{H}_{-}\right), 1 \leq i \leq n$, we define

$$
d F * f:=\left(\begin{array}{c}
\sum_{j=1}^{n} d F_{1 j} * f_{j} \\
\vdots \\
\sum_{j=1}^{n} d F_{n j} * f_{j}
\end{array}\right),
$$

where $d F_{i j}$ denote the measure on $\mathbb{R}$ induced by $F_{i j}$ and $d F_{i j} * f_{j}$ denotes the convolution of the measure $d F_{i j}$ and the function $f_{j}$. If $f$ is continuous on $[-h, \infty)$, then of course

$$
(d F * f)(t)=\int_{0}^{h} d F(\tau) f(t-\tau) \quad \text { for } t \geq 0 .
$$

Consider the retarded system

$$
\begin{aligned}
\dot{x} & =d A * x+B u, \\
y & =C x, \\
x_{\{[-h,(1)]} & =x_{0} \in C\left([-h, 0], \mathbb{F}^{n}\right),
\end{aligned}
$$


where $A \in B V\left([0, h], \mathbb{F}^{n \times n}\right), B \in \mathbb{R}^{n \times m}$, and $C \in \mathbb{F}^{m \times n}$. We assume that

$$
\operatorname{det}(C B) \neq 0
$$

and

$$
\operatorname{det}\left(\begin{array}{cr}
s I-\hat{A}(s) & -B \\
C & 0
\end{array}\right) \neq 0 \text { for all } s \in \overline{\mathbb{C}}_{+},
$$

where $\hat{A}(s):=\int_{0}^{h} \exp (-s \tau) d A(\tau)$ denotes the Laplace-Stieltjes transform of $A$. The transfer function matrix $G(s)$ of $(5.1)$ is given by

$$
G(s)=C(s I-\hat{A}(s))^{-1} B .
$$

Remark 5.1. As in the finite-dimensional case, we shall call (5.3) the minimum-phase condition. It can be shown that (5.3) holds if and only if the following three conditions hold:

(i) The transfer function matrix $G(s)$ has no zeros in $\bar{C}_{+}$;

(ii) $r k(s I-\hat{A}(s), B)=n$ for all $s \in \overline{\mathbb{C}}_{+}$;

(iii) $r k\left(\begin{array}{c}s I-\hat{A}(s) \\ C\end{array}\right)=n$ for all $s \in \overline{\mathbb{C}}_{+}$.

Let $\rho_{i}, \delta_{i}(i=1, \ldots, m), \rho, \delta, p$, and $q$ be as in $\S 4$ and let

$$
\begin{aligned}
& \dot{\xi}=A_{M} \xi+B_{M} v, \quad \xi(0)=\xi_{0} \in \mathbb{E}^{l} \\
& z=C_{M} \xi+I_{m} v
\end{aligned}
$$

be a stabilizable and detectable realization of $M(s)=[q(s) / p(s)] I_{m}$. We shall consider the closed-loop system given by $(5.1),(5.4)$,

$$
\begin{aligned}
& v(t)=k(t) K_{\sigma(k(t))}(y(t)-r(t)), \\
& \dot{k}(t)=\|y(t)-r(t)\|^{2}, \quad k(0)=k_{0} \in \mathbb{R},
\end{aligned}
$$

and

$$
u(t)=z(t)+d(t)
$$

where $r \in \mathcal{S}_{\rho}, d \in \mathcal{S}_{\gamma}+L^{2}\left(\mathbb{H}_{+}, L^{\prime \prime}\right)$ and $K_{1}, \ldots, K_{N}$ and $\sigma: \mathbb{R}:\{1, \ldots, N\}$ are defined as in $\$ 3$.

The following result shows that the universal adaptive controller presented in $\$ 4$ achieves asymptotic tracking and disturbance rejection for the class of retarded systems satisfying (5.2) and (5.3).

THEOREM 5.2. If (5.2) and (5.3) are satisfied, then for any $x_{0} \in C\left([-h, 0], \mathbb{R}^{n}\right), \xi_{0} \in$ ${ }^{\prime \prime}, l, k_{0} \in \mathbb{F}_{-}, r \in \mathcal{S}_{\rho}$, and $d \in \mathcal{S}_{\delta}+L^{2}\left(\mathbb{F}_{+}, w_{-}^{m}\right)$, the closed-loop system given by (5.1) and (5.4)-(5.6) has the following properties:

(i) $\lim _{t \rightarrow \infty} k(t)$ exists and is finite;

(ii) $\left.y-r \in L^{2}\left(\mathrm{jik}_{+},\right]^{m} \mathrm{H}^{m}\right) \cap L^{\infty}\left(\mathbb{F}_{+}, \mathrm{Lu}^{\mathrm{w}}, m\right)$;

(iii) $\lim _{t \rightarrow \infty}(y(t)-r(t))=0$;

(iv) $(x, \xi)^{T} \in L^{\infty}\left(\mathbb{F}_{+}, i^{n+l}\right)$ provided $r$ is bounded.

Proof. First of all it follows from (5.2) and (5.3) that

$$
G^{-1}(s)=s(C B)^{-1}+H(s)
$$

where $H \in H^{\infty}\left(U^{m \times m}\right)$ (see Logemann and Mårtensson [13]), i.e., $G^{-1}(s)$ admits a decomposition of the form (2.1). We proceed in four steps. 
Step 1. Recall from the proof of Theorem 4.1 that

$$
d_{M}(t)=\mathcal{L}^{-1}\left(M^{-1} \hat{d}\right)(t) \in L^{2}\left(\mathcal{L}_{+}, \mathbb{F}^{m}\right)
$$

Defining

$$
\chi(t):= \begin{cases}0, & t=0, \\ 1, & t \in(0, h]\end{cases}
$$

and setting

$$
\begin{aligned}
A_{s e}(\cdot) & :=\left(\begin{array}{cc}
A(\cdot) & \chi(\cdot) B C_{M} \\
0 & \chi(\cdot) A_{M}
\end{array}\right), \quad B_{s e}:=\left(\begin{array}{c}
B \\
B_{M}
\end{array}\right), \\
C_{s e} & :=(C, 0) \text { and } x_{s e}:=\left(\begin{array}{c}
x \\
\xi
\end{array}\right),
\end{aligned}
$$

the series connection of (5.4) followed by $(5.1)$ in the presence of the disturbance $d$ can be reformulated as follows:

$$
\begin{aligned}
\dot{x}_{s e} & =d A_{s c} * x_{s e}+B_{s e}\left(v+d_{M}\right), \\
y & =C_{s e} x_{s e} \\
x_{s e}(t) & =\left(\begin{array}{c}
x_{0}(t) \\
\xi_{0}
\end{array}\right) \quad \text { for all } t \in[-h, 0] .
\end{aligned}
$$

It follows trivially from (5.2) that

$$
\operatorname{det}\left(C_{\text {se }} B_{\text {se }}\right) \neq 0 \text {. }
$$

Moreover, since $q(s)$ is Hurwitz, it follows from the stabilizability and detectability of (5.4) that

$$
\operatorname{det}\left(\begin{array}{rr}
s I-A_{M} & -B_{M} \\
C_{M} & I_{m}
\end{array}\right) \neq 0 \quad \text { for all } s \in \overline{\widetilde{C}}_{+}
$$

Realizing that

$$
\begin{aligned}
& \operatorname{det}\left(\begin{array}{cc}
s I-\hat{A}_{s e}(s) & -B_{s e} \\
C_{s e} & 0
\end{array}\right) \\
& =\operatorname{det}(s I-\hat{A}(s)) \operatorname{det}(G(s)) \operatorname{det}\left(s I-A_{M}\right) \operatorname{det}(M(s)) \\
& =\operatorname{det}\left(\begin{array}{rr}
s I-\hat{A}(s) & -B \\
C & 0
\end{array}\right) \operatorname{det}\left(\begin{array}{rr}
s I-A_{M} & -B_{M} \\
C_{M} & I_{m}
\end{array}\right),
\end{aligned}
$$

we obtain from (5.3) and (5.11)

$$
\operatorname{det}\left(\begin{array}{lc}
s I-\hat{A}_{s e}(s) & -B_{s e} \\
C_{s e} & 0
\end{array}\right) \neq 0 \quad \text { for all } s \in \overline{\mathbb{C}}_{+},
$$

i.e., the series connection of (5.4) followed by (5.1) is minimum-phase.

Step 2. It follows from (5.10) that $\mathbb{F}^{n+1}=\operatorname{ker} C_{s e} \oplus \mathrm{im} B_{s e}$. Hence there exists a non-singular real transformation $P \in \mathbb{E}^{(}(n+l) \times(n+l)$ such that

$$
P^{-1} B_{s e}=\left(\begin{array}{c}
C B \\
0
\end{array}\right), \quad C_{s e} P=\left(\begin{array}{ll}
I_{m} & 0
\end{array}\right)
$$


It is useful to partition the matrix $P^{-1} A_{\text {se }}(\cdot) P$ as follows:

$$
P^{-1} A_{s e}(\cdot) P=\left(\begin{array}{cc}
A_{11}(\cdot) & A_{12}(\cdot) \\
A_{21}(\cdot) & A_{22}(\cdot)
\end{array}\right)
$$

where $A_{11}(\cdot), A_{12}(\cdot), A_{21}(\cdot)$, and $A_{22}(\cdot)$ are matrices with entries in $B V([0, h]$, ].t. of size $m \times m, m \times(n+l-m),(n+l-m) \times m$ and $(n+l-m) \times(n+l-m)$, respectively. Setting $\eta_{s e}(t)=P^{-1} x_{s e}(t)$, it follows from (5.9) that

$$
\begin{aligned}
\dot{\eta}_{s e} & =d\left(P^{-1} A_{s e} P\right) * \eta_{s e}+P^{-1} B_{s e}\left(v+d_{M}\right), \\
y & =C_{s e} P \eta_{s e}, \\
\left.\eta_{s e}\right|_{[-h, 0]} & =\left.P^{-1} x_{s e}\right|_{[-h, 0]} .
\end{aligned}
$$

Since $\eta_{s e}$ can be written as $\eta_{s e}=(y, \eta)^{T}$, it is clear that (5.13) can be decomposed as$$
\dot{y}=C B v_{1},
$$$$
\dot{\eta}=d A_{22} * \eta+d A_{21} * v_{2},
$$$$
\gamma=-(C B)^{-1}\left(d A_{12} * \eta+d A_{11} * v_{2}\right)
$$$$
v_{1}=v+d_{M}-\gamma, \quad v_{2}=y
$$$$
\left.y\right|_{[-h, 0]}=\eta_{1},\left.\quad \eta\right|_{\left[-h_{2}, 0\right]}=\eta_{2},
$$

where $\left(\eta_{1}, \eta_{2}\right)=\left.\eta_{s e}\right|_{[-h, 0]}$ and in particular $\eta_{1}=C x_{0}$. Let $\eta\left(\eta_{2}, \eta_{1}, \omega\right)$ denote the solution of the retarded system (5.15) driven by the initial conditions $\left.\eta\right|_{[-h, 0]}=\eta_{2},\left.v_{2}\right|_{[-h, 0]}=\eta_{1}$ and the input $v_{2} \mid[0, \infty)=\omega \in L L^{2}\left(\mathbb{F}_{+}^{\prime}, \mathbb{E}^{m}\right)$. The corresponding output $\gamma\left(\eta_{2}, \eta_{1}, \omega\right)$ can be written in the form

$$
\gamma\left(\eta_{2}, \eta_{1}, \omega\right)=\mathcal{K} \omega+\tilde{w}
$$

where

$$
\mathcal{K} w=-(C B)^{-1}\left(d A_{12} * \eta(0,0, \omega)+d A_{11} * \omega\right)
$$

and

$$
\tilde{w}=-(C B)^{-1}\left(d A_{12} * \eta\left(\eta_{2}, \eta_{1}, 0\right)+d A_{11} * \eta_{1}\right) .
$$

Step 3. We claim that the retarded system (5.15) is exponentially stable, which is equivalent to saying that $\operatorname{det}\left(s I-\hat{A}_{22}(s)\right) \neq 0$ for all $s \in \overline{\mathbb{C}}_{+}$, where $\hat{A}_{22}(s)=\int_{0}^{h} \exp (-s \tau) d A_{22}(\tau)$ (cf. Hale [6, p. 165]). It follows from the properties of $P$ that

$$
\operatorname{det}\left(\begin{array}{cc}
s I-\hat{A}_{s e}(s) & -B_{s e} \\
C_{s e} & 0
\end{array}\right)=\operatorname{det}\left(\begin{array}{ccc}
s I-\hat{A}_{11}(s) & -\hat{A}_{12}(s) & -C B \\
-\hat{A}_{21}(s) & s I-\hat{A}_{22}(s) & 0 \\
I & 0 & 0
\end{array}\right) .
$$

Defining

$$
T_{1}(s):=\left(\begin{array}{ccc}
I & 0 & -\left(s I-\hat{A}_{11}(s)\right) \\
0 & I & \hat{A}_{21}(s) \\
0 & 0 & I
\end{array}\right), \quad T_{2}(s):=\left(\begin{array}{ccc}
I & 0 & 0 \\
0 & I & 0 \\
0 & -(C B)^{-1} \hat{A}_{12}(s) & I
\end{array}\right),
$$


we obtain

$$
\begin{aligned}
& \operatorname{det}\left(\begin{array}{cc}
s I-\hat{A}_{s e}(s) & -B_{s e} \\
C_{s e} & 0
\end{array}\right) \\
& =\operatorname{det}\left\{T_{1}(s)\left(\begin{array}{ccc}
s I-\hat{A}_{11}(s) & -\hat{A}_{12}(s) & -C B \\
-\hat{A}_{21}(s) & s I-\hat{A}_{22}(s) & 0 \\
I & 0 & 0
\end{array}\right) T_{2}(s)\right\} \\
& =\operatorname{det}\left(\begin{array}{ccc}
0 & 0 & -C B \\
0 & s I-\hat{A}_{22}(s) & 0 \\
I & 0 & 0
\end{array}\right) \\
& =(-1)^{m} \operatorname{det}(C B) \operatorname{det}\left(s I-\hat{A}_{22}(s)\right)
\end{aligned}
$$

Hence $\operatorname{det}\left(s I-\hat{A}_{22}(s)\right) \neq 0$ for all $s \in \overline{\mathbb{U}}_{+}$by the minimum-phase property (5.12).

Step 4. As a consequence of the exponential stability of the retarded system (5.15), the linear mapping $\mathcal{K}$ defined by (5.19) is bounded from $L^{2}\left(\mathbb{F}_{+}, \mathbb{E}^{m}\right)$ into itself and the function $\tilde{w}$ is in $L^{2}\left(\mathbb{P}_{+}, \mathbb{R}^{m}\right)$. Moreover, it is clear that the operator $\mathcal{K}$ is shift-invariant. The system given by $(5.14)-(5.17)$ can be written as

$$
\dot{y}=C B(v-\mathcal{K} y-w), \quad y(0)=C x_{0}(0),
$$

where $w:=\tilde{w}-d_{M} \in L^{2}\left(\mathbb{E}_{+}, \mathbb{E}^{m}\right)$. Let $\mathcal{K}$ be the unique element in $H^{\infty}\left(\tau^{m \times m}\right)$ such that $\mathcal{K}=\mathcal{L}^{-1} \mathcal{M}_{K} \mathcal{L}$. It is easy to see that $K$ is of the form required for the application of Theorem 4.1, i.e.,

$$
K(s)=s\left(\frac{p(s)}{q(s)}-1\right)(C B)^{-1}+\frac{p(s)}{q(s)} H(s),
$$

where $H(s)$ is given by (5.7). Statements (i)-(iii) follow now from Theorem 4.1. Finally, suppose that $r$ is bounded. By statement (ii) this implies that $y$ is bounded, and hence using the exponential stability of (5.15), we see that $\eta$ is bounded. As a consequence $\eta_{\text {se }}=(\eta, y)^{T}$ is bounded, which in turn implies the boundedness of $x_{s e}=(x, \xi)^{T}$.

5.2. Integrodifferential convolution systems. Another interesting class of systems covered by Theorem 4.1 is the class of integrodifferential convolution systems. Consider the system

$$
\begin{aligned}
\dot{x} & =A * x+B u, \\
y & =C x, \\
x(0) & =x_{0} \in \mathbb{F}^{n},
\end{aligned}
$$

where $A \in M\left(\mathbb{F}_{+}^{\prime}, \mathbb{H}_{-}^{n \times n}\right), B \in \mathbb{F}_{*}^{n \times m}$, and $C \in \mathbb{F}_{*}^{m \times n}$. The Volterra integrodifferential system

$$
\begin{aligned}
\dot{x}(t) & =A_{0} x(t)+\int_{0}^{t} A_{1}(t-\tau) x(\tau) d \tau+B u(t), \\
y(t) & =C x(t), \\
x(0) & =x_{0} \in \mathbb{E}^{n},
\end{aligned}
$$

where $A_{0} \in \mathbb{E}_{s}^{n \times n}$ and $A_{1} \in L^{1}\left(\mathbb{F}_{+}^{+}, \mathbb{E}^{n \times n}\right)$ is obviously a special case of (5.22). We assume that

$$
\operatorname{det}(C B) \neq 0
$$


and

$$
\operatorname{det}\left(\begin{array}{cc}
s I-\hat{A}(s) & -B \\
C & 0
\end{array}\right) \neq 0 \quad \text { for all } s \in \overline{\mathrm{C}}_{+},
$$

where $\hat{A}(s):=\int_{0}^{\infty} \exp (-s \tau) d A(\tau)$.

THEOREM 5.3. If (5.23) and (5.24) are satisfied, then for any $x_{0} \in \mathbb{E}^{n}, \xi_{0} \in \mathbb{F}^{\prime}, k_{0} \in \mathbb{F}^{\prime \prime}$, $r \in \mathcal{S}_{\rho}$, and $d \in \mathcal{S}_{\delta}+L^{2}\left(\mathbb{E}_{+}, \mathbb{E}^{m}\right)$, the closed-loop system given by (5.22) and (5.4)-(5.6) has the following properties:

(i) $\lim _{t \rightarrow \infty} k(t)$ exists and is finite;

(ii) $y-r \in L^{2}\left(\mathbb{F}_{+}, \mathbb{R}^{m}\right) \cap L^{\infty}\left(\mathbb{R}_{+}, \mathbb{R}^{m}\right)$;

(iii) $\left.\lim _{t \rightarrow \infty}(y(t))-r(t)\right)=0$;

(iv) $(x, \xi)^{T} \in L^{\infty}\left(\mathbb{H}_{+}, \mathbb{H}^{n+l}\right)$, provided $r$ is bounded.

Proof. Defining

$$
\begin{aligned}
& A_{s e}:=\left(\begin{array}{cc}
A & \delta_{0} B C_{M} \\
0 & \delta_{0} A_{M}
\end{array}\right), \quad B_{s e}:=\left(\begin{array}{c}
B \\
B_{M}
\end{array}\right), \\
& C_{s e}:=(C, 0) \quad \text { and } \quad x_{s e}:=\left(\begin{array}{l}
x \\
\xi
\end{array}\right),
\end{aligned}
$$

where $\delta_{0}$ denotes the unit point mass at 0 , the series connection of (5.4) followed by (5.22) in the presence of the disturbance $d$ can be formulated as follows:

$$
\begin{aligned}
\dot{x}_{s e} & =A_{s e} * x_{s e}+B_{s e}\left(v+d_{M}\right), \\
y & =C_{s e} x_{s e}, \\
x_{s e}(0) & =\left(\begin{array}{c}
x_{0} \\
\xi_{0}
\end{array}\right),
\end{aligned}
$$

where $d_{M}$ is given by (5.8).

Using the same coordinate transformation $P$ as in $\S 5.1$, it is clear that $(5.25)$ can be written in the form

$$
\dot{y}=C B v_{1},
$$

$$
\begin{aligned}
& \dot{\eta}=A_{22} * \eta+A_{21} * v_{2}, \\
& \gamma=-(C B)^{-1}\left(A_{12} * \eta+A_{11} * v_{2}\right)
\end{aligned}
$$

$$
v_{1}=v+d_{M}-\gamma, \quad v_{2}=y,
$$

$$
(y(0), \eta(0))^{T}=P^{-1} x_{s c}(0)
$$

where $(y, \eta)^{T}=P^{-1} x_{s e}$ and the $A_{i j}$ are bounded matrix-valued measures on $\mathbb{F}_{+}$. Let $R$ denote the differential resolvent of the integrodifferential system (5.27), i.e., $R$ is the unique solution of

$$
\dot{R}=A_{22} * R, \quad R(0)=I
$$

The solution $\eta$ is then given by

$$
\eta(t)=R(t) \eta(0)+\left(R * A_{21} * v_{2}\right)(t)
$$


(see Gripenberg, Londen, and Staffans [5, p. 76]) and the output $\gamma$ can be written in the form

$$
\gamma=\mathcal{K} v_{2}+\tilde{w}
$$

where

$$
\mathcal{K} v_{2}=-(C B)^{-1}\left(A_{12} * R * A_{21}+A_{11}\right) * v_{2}
$$

and

$$
\tilde{w}=-(C B)^{-1}\left(A_{12} * R\right) \eta(0) .
$$

Now we can show, as in $\S 5.1$, that

$$
\operatorname{det}\left(s I-\hat{A}_{22}(s)\right) \neq 0 \quad \text { for all } s \in{\overline{\mathbb{C}_{+}}}_{+}
$$

where $\hat{A}_{22}(s)=\int_{0}^{\infty} \exp (-s \tau) d A_{22}(\tau)$, and hence $R$ is integrable (see Gripenberg, Londen, and Staffans [5, p. 83]). It follows that the linear operator $\mathcal{K}$ defined by (5.30) is bounded from $L^{2}\left(\left[\mathbb{H}_{+}, \mathbb{F}^{m}\right)\right.$ into itself. Moreover it is trivial to show that $\mathcal{K}$ is shift-invariant. Since $R$ is integrable we obtain from Gripenberg, Londen, and Staffans [5, p. 83], that the entries of $R$ are square-integrable as well. Therefore the function $\tilde{w}$ defined by $(5.31)$ is in $L^{2}\left(\mathbb{F}_{+}, \mathbb{F}_{+}^{m}\right)$. Finally it follows that the system $(5.25)$ can be written as

$$
\dot{y}=C B(v-\mathcal{K} y-w), \quad y(0)=C x_{0},
$$

where $w:=\tilde{w}-d_{M}$ is in $L^{2}\left(\mathbb{F}_{+}, \mathbb{F}^{m}\right)$ (by (5.8)) and $\mathcal{K}: L^{2}\left(\mathbb{F}_{+}, \mathbb{F}^{m}\right) \rightarrow L^{2}\left(\mathbb{R}_{+}, \mathbb{F}^{m}\right)$ is linear bounded and shift-invariant. The claim now follows in exactly the same way as in the proof of Theorem 5.2.

6. Conclusions. In this paper we have presented an input-output approach to the adaptive servoproblem for multivariable infinite-dimensional minimum-phase systems with invertible high-frequency gains. In particular, we have shown the following:

- The switching algorithm, introduced by Byrnes and Willems [1] in a finite-dimensional state-space set-up, stabilizes any infinite-dimensional plant belonging to the class of systems given by (2.1).

- The series interconnection of the Byrnes-Willems controller with a suitable precompensator solves the adaptive servoproblem for the class of systems satisfying (2.1).

- The input-output results obtained in $\$ \S 3$ and 4 apply to retarded systems and integrodifferential convolution systems.

The adaptive control laws presented in $\$ \S 3$ and 4 give positive answers to feasibility and existence questions. They do not provide satisfying adaptive controllers from an engineer's point of view. However, the following comments show that the results of this paper might also be of some practical importance.

- It seems plausible that the technique in $\$ 4$ (or variations thereof) can be used in order to obtain adaptive servomechanisms from various adaptive stabilization algorithms available in the literature.

- If the conjecture formulated in $\S 3$ turns out to be true, the high-gain switching algorithm can be used in order to identify a stabilizing linear controller or a linear servocompensator for the class of infinite-dimensional systems under consideration by a single simulation. 


\section{Appendix.}

Proof of Lemma 3.2. The closed-loop system is given by

$$
\begin{aligned}
& \dot{y}(t)=D\left(k(t) K_{\sigma(k(t))} y(t)-(\mathcal{H} y)(t)-w(t)\right), \\
& \dot{k}(t)=\|y(t)\|^{2}, \quad t \geq 0, \\
& y(0)=y_{0}, \quad k(0)=k_{0} .
\end{aligned}
$$

Without loss of generality we may assume that $k_{0} \geq 0$. The proof is divided into three steps.

Step 1. Existence and uniqueness on a "small" interval.

Consider equation (7.1) with $\sigma(k(t))$ replaced by $\sigma\left(k_{0}\right)$, i.e.,

$$
\begin{aligned}
& \dot{y}(t)=D\left(k(t) K_{\sigma\left(k_{0}\right)} y(t)-(\mathcal{H} y)(t)-w(t)\right), \\
& \dot{k}(t)=\|y(t)\|^{2}, \quad t \geq 0, \\
& y(0)=y_{0}, \quad k(0)=k_{0}
\end{aligned}
$$

By Theorem $2.5,(7.2)$ has a unique absolutely continuous solution $(\tilde{y}, \tilde{k})$ on some interval $[0, T)$. Set $\tau\left(k_{0}\right)=\min \left\{\tau_{i} \mid \tau_{i}>k_{0}\right\}$ and let $T^{\prime} \in(0, T)$ be such that $\tilde{k}\left(T^{\prime}\right)<\tau\left(k_{0}\right)$. Since $\sigma(\hat{k}(t))=\sigma\left(k_{0}\right)$ for all $t \in\left[0, T^{\prime}\right]$, it follows that $(\tilde{y}, \tilde{k})$ is the unique solution of (7.1) on $\left[0, T^{\prime}\right)$.

Step 2. Extended uniqueness.

Let $\left(y_{i}, k_{i}\right)$ be solutions of $(7.1)$ on $\left[0, T_{j}\right), i=1,2$. We claim that $\left(y_{1}, k_{1}\right) \equiv\left(y_{2}, k_{2}\right)$ on $[0, T)$, where $T:=\min \left(T_{1}, T_{2}\right)$. Let us assume the contrary, i.e. there exists $t \in(0, T)$ for which $\left(y_{1}(t), k_{1}(t)\right) \neq\left(y_{2}(t), k_{2}(t)\right)$. Defining

$$
t^{*}:=\inf \left\{t \in(0, T) \mid\left(y_{1}(t), k_{1}(t)\right) \neq\left(y_{2}(t), k_{2}(t)\right)\right\},
$$

it follows that $t^{*}>0$ (by Step 1) and $\left(y_{1}\left(t^{*}\right), k_{1}\left(t^{*}\right)\right)=\left(y_{2}\left(t^{*}\right), k_{2}\left(t^{*}\right)\right)$ (by continuity). Now set $k^{*}:=k_{1}\left(t^{*}\right)=k_{2}\left(t^{*}\right)$ and realize that the initial-value problem

$$
\begin{aligned}
\dot{y}(t) & =D\left(k(t) K_{\sigma\left(k^{*}\right)} y(t)-(\mathcal{H} y)(t)-w(t)\right), \\
\dot{k}(t) & =\|y(t)\|^{2}, \quad t \geq t^{*}, \\
\left.y\right|_{\left[0, t^{*}\right]} & =\left.y_{1}\right|_{\left[0, t^{*}\right]},\left.\quad k\right|_{\left[0, t^{*}\right]}=\left.k_{1}\right|_{\left[0, t^{*}\right]}
\end{aligned}
$$

is solved by $\left(y_{1}, k_{1}\right)$ and $\left(y_{2}, k_{2}\right)$ on $\left[0, t^{*}+\epsilon\right)$ for some sufficiently small $\epsilon>0$. It follows from Theorem 2.5 that $\left(y_{1}(t), k_{1}(t)\right)=\left(y_{2}(t), k_{2}(t)\right)$ for all $t \in\left[0, t^{*}+\epsilon\right)$, which contradicts the definition of $t^{*}$.

Step 3. Continuation of solutions.

Let $(\tilde{y}, \tilde{k})$ be a solution of $(7.1)$ on $[0, T), 0<T<\infty$. Assume that $(\tilde{y}, \tilde{k})$ is bounded. We claim that under these conditions the solution $(\tilde{y}, \tilde{k})$ can be continued to the right (beyond $T$ ). Since $\tilde{k}$ is bounded, continuous, and nondecreasing, it is clear that $\lim _{t \rightarrow T} \tilde{k}(t)=: \tilde{k}_{T}$ exists and is finite. As a consequence we have $\tilde{y} \in L^{2}\left(0, T ; \mathbb{R}^{m}\right)$ and hence, by (7.1), $\dot{\tilde{y}} \in L^{2}\left(0, T ; \mathbb{Z}^{m}\right) \subset L^{1}\left(0, T ; \mathbb{F}^{m}\right)$. Using the fact that

$$
\tilde{y}(t)=y_{0}+\int_{0}^{t} \dot{\tilde{y}}(\tau) d \tau,
$$

it follows that $\lim _{t \rightarrow T} \tilde{y}(t)=: \tilde{y}_{T}$ exists and is finite. By Theorem 2.5 the initial-value problem

$$
\begin{aligned}
& \dot{y}(t)=D\left(k(t) K_{\sigma\left(\tilde{k}_{T}\right)} y(t)-(\mathcal{H} y(t)-w(t)),\right. \\
& \dot{k}(t)=\|y(t)\|^{2}, \quad t \geq T, \\
& y(t)=\left\{\begin{array}{ll}
\tilde{y}(t), & t \in[0, T), \\
\tilde{y}_{T}, & t=T,
\end{array} \quad k(t)= \begin{cases}\tilde{k}(t), & t \in[0, T), \\
\tilde{k}_{T}, & t=T,\end{cases} \right.
\end{aligned}
$$


has a unique absolutely continuous solution $(\bar{y}, \bar{k})$ on $[0, T+\epsilon)$ for some $\epsilon>0$. Finally let $\delta \in(0, \epsilon)$ be such that

$$
\bar{k}(T+\delta)<\min \left\{\tau_{i} \mid \tau_{i}>\tilde{k}_{T}\right\}
$$

Then $(\bar{y}, \bar{k})$ is a solution of $(7.1)$ on $[0, T+\delta)$ extending the solution $(\tilde{y}, \tilde{k})$.

Acknowledgments. A substantial part of the research for this paper was done while $\mathrm{H}$. Logemann was visiting the Department of Mathematics and the School of Engineering at the University of Exeter. A. Ilchmann was supported by the Deutsche Forschungsgemeinschaft and the University of Exeter.

\section{REFERENCES}

[1] C. I. Byrnes And J. C. Willems, Adaptive stabilization of multivariable linear systems, Proc. 23rd IEEE Conference on Decision and Control, Las Vegas, NV, 1984, pp. 1574-1577.

[2] F. M. CAllier AND C. A. Desoer, Stabilization, tracking and disturbance rejection in multivariable convolution systems, Ann. Soc. Sci. Bruxelles, 94 (1980), pp. 7-51.

[3] R. F. CURTAin, Tracking and regulation for distributed parameter systems, Matematica Aplicada E Computacional, 2 (1983), pp. 199-218.

[4] B. A. FRANCIS, The multivariable servomechanism problem from the input-output viewpoint, IEEE Trans. Automat. Control, AC-22 (1977), pp. 322-328.

[5] G. Gripenberg, S.-O. Londen, and O. Staffans, Volterra Integral and Functional Equations, Cambridge University Press, Cambridge, UK, 1990.

[6] J. Hale, Theory of Functional Differential Equations, Springer-Verlag, New York, 1977.

[7] C. J. Harris and J. M. E. ValenCA, The Stability of Input-Output Dynamical Systems, Academic Press, London, 1983.

[8] U. Helmke, D. PrÄtzel-Wolters, and S. Schmid, Sufficient conditions for adaptive stabilization and tracking, Bericht 38 der Arbeitsgruppe Technomathematik, Fachbereich Mathematik, Universität Kaiserslautern, Kaiserslautern, Germany, 1989.

[9] - Adaptive tracking for scalar minimum-phase systems, in Control of Uncertain Systems, D. Hinrichsen and B. Mårtensson, eds., Birkhäuser, Boston, 1990, pp. 101-117.

[10] D. Hinrichsen and A. J. Pritchard, Destabilization by output feedback, Differential Integral Equations, 5 (1992), pp. 357-386.

[11] A. Ilchmann And H. Logemann, High-gain adaptive stabilization of multivariable systems-revisited, Systems Control Lett., 18 (1992), pp. 355-364.

[12] H. Logemann, Funktionentheoretische Methoden in der Regelungstheorie undendlichdimensionaler Systeme, Ph.D. thesis, Institut für Dynamische Systeme, Universität Bremen, Bremen, Germany, 1986 (Report 156).

[13] H. Logemann and B. MÅrtensson, Adaptive stabilization of infinite-dimensional systems, IEEE Trans. Automat. Control, AC-37 (1992), pp. 1869-1883.

[14] H. LOGEMANN AND D. H. OwEns, Input-output theory of high-gain adaptive stabilization of infinite-dimensional systems with non-linearities, Internat. J. Adaptive Control Signal Processing, 2 (1988), pp. 193-216.

[15] H. Logemann AND H. Zwart, On robust PI-control of infinite-dimensional systems, SIAM J. Control Optim., 30 (1992), pp. 573-593.

[16] I. MAREELS, A simple selftuning controller for stably invertible systems, Systems Control Lett., 4 (1984), pp. 5-16.

[17] B. MÅrtensSon, Adaptive stabilization, Ph.D. thesis, Lund Institute of Technology, Dept. of Automatic Control, Lund, Sweden, 1986.

[18] —-, Adaptive stabilization of multivariable systems, Contemp. Math., 68 (1987), pp. 191-225.

[19] - Adaptive stabilization without high-gain, in Identification and Adaptive Control, C. I. Byrnes and A. Kurzmansky, eds., Springer-Verlag, Berlin, 1988, pp. 226-238.

[20] D. E. Miller AND E. J. Davison, The self-tuning robust servomechanism problem, IEEE Trans. Automat. Control, AC-34 (1989), pp. 511-523.

[21] - Adaptive control of a family of plants, in Control of Uncertain Systems, D. Hinrichsen and B. Mårtensson, eds., Birkhäuser, Boston, 1990, pp. 197-219.

[22] A. S. MoRSE, A 4 $(n+1)$-dimensional model reference adaptive stabilizer for any relative degree one or two, minimum-phase system of dimension $n$ or less, Automatica, 23 (1987), pp. 123-125.

[23] W. Rudin, Real and Complex Analysis, 2nd ed., McGraw-Hill, New York, 1974.

[24] E. P. RYAN, Adaptive stabilization of multi-input nonlinear systems, Int. J. Robust Nonlinear Control, 3 (1993), pp. 169-181. 
[25] S. B. TOWNLEY, Topological aspects of universal adaptive stabilization, preprint, 1992.

[26] M. VidYASAGar, A note on time-invariance and causality, IEEE Trans. Automat. Control, AC-28 (1983), pp. 929-931.

[27] Control Systems Synthesis: A Factorization Approach, MIT Press, Cambridge, MA, 1985.

[28] M. Vidyasagar, H. SChneIDER, ANd B. A. Francis, Algebraic and topological aspects of feedback stabilization, IEEE Trans. Automat. Control, AC-27 (1982), pp. 880-894.

[29] G. WEISs, Representation of shift invariant operators on $L^{2}$ by $H^{\infty}$ transfer functions: an elementary proof, a generalization to $L^{p}$ and a counterexample for $L^{\infty}$, Math. Control Signals Systems, 4(1991), pp. 193-203.

[30] W. M. Wonham, Linear Multivariable Control: A Geometric Approach, 2nd, ed., Springer-Verlag, New York, 1979.

[31] X.-J. ZHU, A finite spectrum unmixing set for $G L(3, \mathbb{B})$, in Computation and Control, K. Bowers and J. Lund, eds., Birkhäuser, Boston, 1989, pp. 403-410. 